\title{
Advances in simulation-driven optimization and modeling
}

\author{
Slawomir Koziel ${ }^{\mathrm{a}, *}$, Leifur Leifsson ${ }^{\mathrm{a}}$ and Xin-She Yang ${ }^{\mathrm{b}}$ \\ ${ }^{a}$ Engineering Optimization and Modeling Center, School of Science and Engineering, Reykjavik \\ University, Reykjavik, Iceland \\ ${ }^{\mathrm{b}}$ Mathematics and Scientific Computing, National Physical Laboratory, Teddington, UK
}

Computer simulations are ubiquitous in contemporary engineering and science. In numerous fields, including mechanical engineering, civil engineering, electrical engineering, structural and aerospace engineering, automotive industry, oil industry, chemical engineering, ocean science and climate research to name just a few, simulation plays a critical role not only for verification purposes, but, more importantly in the design process itself. The complexity of structures and systems makes it analytically intractable, and it is thus extremely time-consuming and challenging to carry out any realistic design tasks, and in many cases, it is almost impossible to achieve any sensible design solutions under stringent constraints. These challenging tasks can be to optimally adjust the geometry and/or material parameters so that the system meets given performance requirements, or to calibrate the model parameters to make it fit given measurements, or to generate the optimal paths/routes for scheduling and planning tasks.

In most cases, the interactions can be highly complex and multifold, and it is not easy or possible to isolate the processes of interest in the simplest, solvable form. For example, in the design of an electronic device, it is not just the isolated device to be designed that needs to be considered but also its - sometimes complex - interactions with the environment that affect the device's performance. On the other hand, using accurate, realistic simulations allows the engineers to avoid costly prototyping and to realize the design closure with numerical models rather than through physical system measurements and prototype re-building. Furthermore, accurate simulations make it possible to analyze phenomena that could not be captured using simplistic theoretical models or too expensive or too time-consuming to be investigated through physical measurements.

While high-fidelity numerical models can be very accurate, they tend to be computationally expensive. Simulation times of several hours, days, or weeks are not uncommon. In many cases, it may be a highly challenging task to just set up the model that takes into account all main, relevant system components and their interactions. One of the consequences is that a direct use of high-fidelity simulations in the optimization process may be prohibitive. The presence of massive computing resources is not always translated into computational speedup in practice, which is due to a growing demand for simulation

\footnotetext{
${ }^{*}$ Corresponding author: Slawomir Koziel, Engineering Optimization and Modeling Center, School of Science and Engineering, Reykjavik University, Reykjavik, Iceland. E-mail: koziel@ru.is.
} 
accuracy, both by including multi-physics and second-order effects, and by using finer discretization of the structure under consideration. As conventional optimization algorithms (e.g., gradient-based schemes with numerical derivatives) require tens, hundreds or even thousands of objective function calls per run, depending on the number of design variables, the computational cost of the whole optimization process is often unacceptably high.

However, computational cost is not the only issue: in many cases simulation results contain numerical errors and noise, which may be derived from using adaptive meshing technique and others. As a result, objective functions coming from computer simulations do not have any explicit form, and consequently analytically intractable. In addition, such objectives can be discontinuous and non-differentiable. On the other hand, sensitivity information is frequently unavailable, or too expensive to compute. In some cases it is possible to obtain derivative information inexpensively through adjoint sensitivities; however, numerical noise is an important issue that can complicate simulation-driven design. Moreover, adjoint sensitivity is an invasive technique (requires detailed knowledge of and access to the source codes of the simulator), and thus cannot be assumed to be generally available, although it is supported by a growing number of commercial simulation packages.

In almost all situations, uncertainties always present in the system parameters of interest. In particular, material properties and geometry of the manufactured device may differ from their nominal values as a result fabrication tolerances. Therefore, the optimization process may seek for the robust design which ensures the highest probability of satisfying the performance requirements under the presence of uncertainties, rather than just for the optimal design.

The aforementioned difficulties make it necessary to search for optimization techniques that are computationally efficient (i.e., reduce the number of CPU-intensive simulations during the optimization run as much as possible), robust (e.g., are able to handle noisy and discontinuous objective functions), and reliable (in particular, have good convergence properties), but also simple enough to implement so that they can be easily acquired by engineers and designers, especially those who want to interface them with various commercial simulation software packages. In this context, the development of efficient and accurate modeling methodologies is of fundamental importance, as fast and smooth models are key components to reduce computational cost in the whole design process.

There has been substantial research effort towards the development of efficient optimization and modeling techniques. These include surrogate-based optimization (SBO) methodologies, where the direct optimization of the computationally expensive model is replaced by an iterative process that involves the creation, optimization and updating of fast and analytically tractable surrogate models that both function-approximation- and physically-based. Other approaches include various derivativefree optimization techniques, e.g., different variants of pattern search algorithm, filtering methods or metaheuristics (e.g., genetic algorithms and particle swarm optimizers). Meta-modeling techniques are another large area of research that aim at constructing local or quasi-global response surfaces surrogate of nice properties (in particular, fast and smooth) using the sampled data from the original, high-fidelity model. A number of meta-modeling techniques have been developed, including radial basis function interpolation, kriging, moving least-squares, artificial neural networks, support vector regression, etc. Substantial progress has been made in the use of adjoint sensitivities, which are now more and more commonly used in various fields of engineering and science, as well as available through a growing number of commercial simulation software packages.

Despite of the progress in the development of techniques for computationally expensive engineering design problems, a number of issues still remain open. The International Workshop on Advances in Simulation-Driven Optimization and Modeling (ASDOM 2011) that took place in Reykjavik, Iceland, in 
August 2011 focused on reviewing the state-of-the-art engineering modeling and optimization techniques, provided a forum for cross-disciplinary discussions, as well as promoting important topics for the future research and development. This special issue of Journal of Computational Methods in Science and Engineering includes several papers selected from and based on the presentations given during ASDOM 2011. These papers cover various aspects of the field, including new algorithms, design automation, simulation-driven optimization and modeling techniques, as well as applications in various areas of science and engineering.

The special issue opens with the paper by Buhren et al. that discusses adaptive and hierarchical metamodeling approach using radial basis functions (RBFs). The authors discuss various techniques of developing the RBF metamodel through suitable infill criteria as well as domain partitioning where separate models are constructed for disjoint sub-domains with a smooth transition between them. This methodology facilitates the construction of the RBF models when a large number of training points have to be considered and the data exhibits different behavior in various regions of the design space.

In the second paper, Parr et al. consider various statistical infill criteria for kriging metamodeling techniques applied for constrained optimization. In their approach, objective improvement and constraint satisfaction are separate goals, treated using multiobjective optimization. The authors demonstrate by selecting model update points in close proximity to the constraints boundaries the regions that are likely to contain the feasible optimum can be better modeled. This may be advantageous when compared to conventional approaches where the constraints are handled through penalty functions, which tend to create multimodal landscapes that are difficult to search.

In the next paper, Priess et al., describe calibration of marine ecosystem model parameters using surrogate-based optimization. The authors consider two types of ocean models: one- and threedimensional describing concentration of various tracers as functions of time. The goal - fitting the model responses to give measurement data - is formulated as a nonlinear optimization problem. Surrogatebased approach exploiting low-fidelity physics-based models is used in order to reduce the computational cost of the process. The authors demonstrate the combining the knowledge about the systems under consideration with simple multiplicative response correction results in yielding satisfactory calibration at a very low computational cost corresponding to several evaluations of the high-fidelity ocean models of interest.

The paper by Marheineke et al. focuses on the application of space mapping optimization for transport processes coming from the fields of fluid dynamics, semiconductors, and radiation. They present model hierarchies and surrogates for each transport process, considering the appropriate physical, algebraic, or grid-based formulations. In each case, the space mapping algorithm compares favorably with the gradient-based fine model optimizations.

In the next paper, Koziel and Leifsson discuss simulation-driven shape optimization with SBO and computational fluid dynamic (CFD) models. They review several design methodologies exploiting lowfidelity CFD models obtained through coarse-discretization and relaxed convergence criteria, as well as response correction techniques that aim at constructing reliable surrogate models. Applications examples, involving the design of axisymmetric hulls in subsonic flow, and airfoils in both subsonic and transonic flows, are presented. Their findings show that significant savings in computational cost can be achieved when compared to the direct optimization of the high-fidelity CFD models.

The paper by Gray et al. deals with methods for the calibration of computational models to improve their accuracy and predictive capabilities. Typically, the model responses are compared with experimental data. In this process, uncertainty quantification (UQ) techniques are used to identify, characterize, reduce, and, if possible, eliminate uncertainties. The authors review basic approaches for model calibration 
and uncertainty quantification. The current methods are serial approaches and can be computationally expensive. The authors then propose an efficient approach for the joint model calibration and uncertainty quantification that combines Bayesian statistical models and derivative-free optimization.

The paper by Yeomans provides a co-evolutionary approach of combining computationally efficient simulation with evolutionary optimization algorithms, and this simulation-driven approach can be used to generate multiple policy alternatives in waste management facility expansion planning. One of the challenges in decision-making is that the final decisions must be constructed not only upon clearly articulated and modeled objectives, but also upon environmental, political and socio-economic goals that are fundamentally subjective. Therefore, the relevant optimization task may not be well-defined in many cases. In this study, the modeling-to-generate alternatives approach is based on the concept of co-evolution and is designed to generate a small number of good but maximally different alternatives, which can help decision-makers to make cost-effective choices and planning.

In the last paper, Yang explores the effectiveness of metaheuristic algorithms for solving inverse problems. In essence, an inverse problem can be considered as an optimization problem because the aim of inversion is to estimate the unknown parameters so as to minimize the differences between the predicted results and the observations. By using the cuckoo search (CS) algorithm, this study shows that CS is also very efficient for inverse modeling. As metaheuristic algorithms are stochastic and adaptive, they can be used to solve a wide variety of problems such as nonlinear global optimization, inversion, and topological optimization.

The guest co-editors would like to thank our authors and contributors for their outstanding contributions to this special issue. We would also like to take this opportunity to acknowledge the effort of many reviewers who helped us shape the contents of this volume. 